\title{
Index of Contributors and Presentations
}

Boldface denotes a presenting author and the number of the abstract being presented.

\section{Author: Presentation}

\section{A}

Agnol MD: P-070

Agra C: P-074, P-108, P-122, P-135

Aguiar LS: P-068

Ah Won L: P-003

Aihara T: A-36

Ajit D: P-044, P-175

Ajjimakorn S: P-153

Akl M: P-149

Aksoy F: P-145

Ali BA: P-139

Ali JIL-20

Alkuwari E: A-12

Allamani M: P-098

Allegretta M: P-087

Allen K: CY-04

Almonte M: A-02

Al-Rawi K: P-136

Alvarez AL: A-31

Amano S: A-32, P-150

Amendoeira I: SS-06

Amin T: P-149

Angel J: P-025, P-112

Anjos S: P-046, P-156

Anttila A: P-155

Aoki D: SS-18

Apilugsanachit A: P-153

Arai Y: SS-16

Aratake Y: AW-01

Ardizzoni A: A-57

Arias V: P-014

Armenti C: IL-06

Arola J: P-028

Arora V: P-047

Ash A: A-24

Ashida Y: P-015

Ashton N: P-079

Aufiero P: P-130

Auger M: A-12, SS-09

Axelson G: P-161

\section{B}

Bacic R: P-048

Badilas C: P-112

Bailey A: A-02

Bakhos S: A-17

Ballet S: A-42

Ban S: P-034

Banerjee D: A-19, IL-19

Barbetakis N: P-025

Barkan GA: A-17, A-31, P-109

Baross A: IL-20

Batoroev Y: P-134

Bazulina LM: P-119
Beccati MD: A-16, A-43

Behtash N: P-045

Belak R: A-19

Bennett I: A-37

Benoit J: P-050

Benoy I: P-157

Bergara S: P-096

Bergeron C: A-08

Bergman S: P-123

Bertini A: P-046, P-156

Bicen B: P-093

Bilbao F: P-096

Bin C: P-114

Birol I: IL-20

Bischiniotis T: P-025

Bjerregaard B: A-20, A-26

Blenc A: A-01

Bobua I: P-127

Boccardo E: A-41

Boer R: A-27, A-30, P-182, P-181

Boerner S: A-50, A-70

Bogatyrev VN: P-004, P-054, P-119

Bogdanska M: A-61, P-138

Bogers J: A-06, A-64, P-157

Bogers JPM: A-68

Boglou K: P-112, P-165:

Bogovskaja J: P-146

Bolliger CT: A-60, P-100

Bonfiglio T: SS-25

Bongiovanni M: A-18

Boon L: A-04, A-05:

Boon M: A-04, A-05, A-07, A-67, A-68

Borrajero Martinez I: SS-29

Bosdet I: IL-20

Boulet G: A-06, P-157

Bowditch R: WK-06

Bozzetti C: A-57

Branca M: A-45

Brinkmann-Smetanay F: P-162

Brisse H: A-42

Broemeling D: A-19

Brown K: A-19

Brundyn K: A-60, A-62, P-100

Bubendorf L: IL-32, SS-08

Buriani C: A-16, A-43

Burzio L: A-41

Burzio V: A-41

C

Cabala A: A-48

Cakalir C: P-033

Cantasdemir M: P-033, P-145

Caraway N: WK-10

Carydis VB: P-158
Cas F: A-08

Casadio C: P-007

Casco C: A-18

Cazzaniga M: P-007

Centeno B: IL-27

Cevlik F: P-111

Chan Kwon J: P-003

Chan N: P-022

Chan S: IL-13, IL-20

Chang-Suk K: P-114, P-115

Charalampidis C: P-112

Chatt G: A-31

Chatzistamou I: A-58

Chau T: P-022

Che Dy: P-006, P-154

Chen WY: P-057

Cheng TY: P-008

Cherian S: P-047

Cheung A: SS-20

Chiapparini L: P-007

Chin-Cheng L: P-059

Chinoy R: P-044, P-128, P-175

Chiu R: IL-20

Chiung-Ju C: P-059

Cho SY: P-144

Choi C: P-001

Choi HJ: P-009

Choi JS: P-062

Choi MS: P-010, P-011

Choi YD: P-001, P-089

Chopin L: A-37

Chosia M: P-073

Choudhury M: P-159

Chun YK: P-062

Chung PF: P-008

Cibas E: IL-18, SS-24

Ciolino A: P-121

Ciotti M: A-45

Colgan TJ: P-158

Company M: P-184

Company MM: P-075

Connors J: IL-20

Conz C: P-046, P-156

Corbett R: IL-20

Costa S: A-45

Couturier J: A-42

Cunha Santos G: A-50

\section{D}

Daa T: P-133

D'all Agnol M: P-067, P-068

Daneshbod Y: P-090, P-110

Danic D: P-137

Dara A: P-098 
Dargent JL: P-036

Das DK: P-139

Davey E: IL-13

Davies P: IL-09

De Boeck G: P-073

de Bosschere M: A-05

de Lelis Feres C: P-067

Dejmek A: A-33, A-40, P-035, SS-19

del Rio MJ: P-173

Delaney A: IL-20

Dellatre O: A-42

Dendukuri N: A-12

Denton K: IL-12

Depuydt C: A-64, P-157:

Desai M: A-02, P-024, P-026, P-079, SS-03, WK-09

Destouni C: P-025, P-038, P-086, P-112, P-165

Di Bonito L: A-45

Di Bonito P: A-45

di Loreto C: P-070

Diacon A: A-60, A-62, P-100

Dighe S: P-175

Dimasis N: P-038

Dolz C: P-184

Domagala-Kulawik J: A-61, P-138

Doudkine A: P-002

Dowie R: A-02

Dubb M: P-083

Dudding N: A-23, A-24, SS-02

Duggan M: A-29, WK-08

Duineveld SM: A-68

Dumonceau JM: A-18

Durak H: P-033

Dvorackova J: P-185

\section{E}

Edgar Z: P-081

El Ganzoury H: P-149

El Lethy T: P-149

Elishaev E: A-48

Elshaer M: P-160

El-Shinnawy I: P-161

English J: IL-24

Enomoto Y: P-020

Erozan Y: AW-05, WK-07

Ersoz C: P-093, P-111

Eschenbach D: A-08

Essink-Bot L: A-27, A-30, P-182

Essink-Bot M: P-181, P-168

Etilinger D: P-068, P-067

Eun Sun J: P-003

Evagelinos D: P-165

Evaggelou I: A-65

Evans M: A-09, A-38, P-087

\section{$\mathbf{F}$}

Fait T: P-053

Fakor F: P-045

Färkkilä M: P-028

Farnsworth A: IL-13

Favalli C: A-45

Fazzito Ipar M: A-18

Fernandez F: P-171, P-172

Fernández L: SS-32

Field M: IL-20
Filho À: P-014

Follen M: IL-15

Franciosi V: A-57

Freitas M: P-048

Fréneaux P: A-42

Friedlander M: A-21

Fujii T: SS-18

Fujikane T: A-56 P-094

Fujioka K: A-22, A-39, P-082, P-097, P-102, P-104, P-105, SS-15

Fujita M.: P-061

Fujita Y: A-56, P-094

Fujiuchi S: A-56, P-094

Fujiwara H: P-088

Fujiwara Y: P-013

Fustar-Preradovic LJ: P-076, P-137

\section{G}

Gaete F: A-41

Gamachi A: P-133

Ganjei-Azar P: A-69

Garcia-Bonafe MM: P-075, P-184

Garvin A: P-123

Gascoyne R: IL-20

Gasparro D: A-57

Garnis C: IL-22

Gatscha R: A-21

Gattuso P: A-17

Gaucher A: P-050

Geddie W: A-50, A-70, SS-11, WK-11

Genevay M: A-18

George SS: P-139

Gerasimidou D: P-038

Ghaemmaghami F: P-045

Ghossein R: P-141

Ghoubari FE: A-07, A-68

Gillenwater A: IL-15

Giorgadze T: P-161, P-051

Giorgi C: A-45

Gissmann L: IL-01

Godyn J: P-130

Gomes L: P-048

Gonzalez M: P-171, P-172, P-173

Gopolang F: A-52

Gorad BD: P-128

Gornicka B: A-61, P-138

Goulart R: P-087

Gregoire J: A-29

Griesser H: IL-26, P-162

Grigoruk OG: P-004, P-119

Guazzi A: A-57

Guggenheimer K: A-19

Guillard M: IL-23

Guimaraes G: P-048

Gunn D: A-19

Gyeongsin P: P-114

Gyeong-Sin P: P-115

H

Haba R: P-042, P-095

Habbema D: A-27, A-30, P-181, P-182

Hachisuga T: P-060, P-088

Hadengue A: A-18

Hájek Z: P-053

Haji BE: P-139

Hakama M: P-155
Halbauer M: P-137, P-076

Haller A: P-036

Halme L: P-028

Hammam O: P-149

Handa U: P-029

Hanrath C: A-03

Hansson M: A-40

Haraoka S: A-46

Harper D: IL-02

Harris J: A-37

Harvey M: A-09

Hashi A: P-169

Hata K: A-59

Hayes M: A-28, A-49, IL-34

Hazra, A: P-130

Herbert A: SS-01

Herington A: A-37

Hermosa C: P-096

Hibbert A: WK-01

Hideki H: P-049

Hilfrich R: A-03, A-67, IL-26

Hirai Y: SS-16, SS-17

Hirakawa E: A-59, P-042, P-095

Hirano T: A-22, A-39, P-082, P-097, P-102, P-103, P-104, P-105, SS-15

Hirao N: SS-18

Hirata S: P-169

Hiroi S: P-085

Hirokawa M: A-11

Hiroko HI: P-077

Hirose T: A-56, P-094

Holloway P: A-67

Holloway-Brons PA: A-68

Holt R: IL-20

Homenuke M: A-19

Honda H: A-22, A-39, P-082, P-097, P-102, P-103, P-104, P-105

Honeyman J: P-173

Hong SW: P-037

Hong SR: P-062

Horai T: A-55

Horsman D: IL-20

Horvath C: A-06, P-157

Hoshi K: P-169

Hoshi R: A-55

Hosone M: P-116

Huang MS: P-008

Huettner P: A-44

Huguet P: P-075

Hulman G: P-164

Hyder P: P-024

I

Ibarrola R: P-096

Ikebata K: SS-16

Ikeda N: P-097

Illingworth A: A-01

Inaniwa Y: A-32

Inoue A: P-061

Inoue M: P-183

Irwig L: IL-13

Ishii Y: P-041

Ishikawa Y: A-55

Ito Sakai Y: P-067

Itoh H: SS-12

Iwai M: P-150 
Iwasaka T: P-072

Iwashita A: A-46

J

Jan IS: P-008

Jandova A: A-53

Javier D: IL-15

Jerse M: P-002

Jin S-Y: P-011, P-010

Jirasek JE: P-179

Johns D: A-23

Johnson L: IL-20

Johnson N: IL-20

Jones A: A-37

Jones S: IL-20, IL-21

Jordan B: P-162

Joshi D: P-047

Juhng SW: P-089

Junaid TA: P-139

Jung JH: P-009

Jung SM: P-078

Jylling AMB: P-012

K

Kobayashi TK: A-47

Kadota K: P-042, P-095

Kagabu T: P-052

Kagiya T: P-023

Kaic G: P-131

Kakihana M: A-22, A-39, P-082, P-102, P-103, P-104, P-105, SS-15

Kaku T: P-072

Kakudo K: P-023

Kalghatgi A: P-084

Kamihara Y: A-46

Kamimori A: SS-17

Kaminsky D: IL-34

Kamoshida S: P-152

Kampas L: P-025, P-038, P-086, P-112, P-165

Kanbour A: A-48

Kanbour-Shakir A: A-48

Kanda M: P-015

Kandil D: P-087

Kanellis G: A-58, A-65

Kang SJ: P-009

Kano M: P-039

Kapila K: P-139

Kaplanis K: P-165

Kardari M: P-032, P-170

Kashima K: P-133

Kashimura M: P-060, P-088

Kashimura Y: P-088

Katalenic S: P-055

Kataoka H: P-150

Katayama H: P-013, P-116, P-151

Kato H: A-22, A-39, P-082, P-097, P-102, P-103, P-104, P-105, SS-15

Katoh R: P-041, P-169

Katsuki N: P-095

Katz R: WK-10

Kaur G: P-019, P-177

Kawagoe T: P-060, P-088

Kawai K: P-152

Kawai T: P-085

Kawamura J: P-152
Kawana T: P-174

Kawashima K: P-034

Kayoko KH: P-077

Keramzadeh M: P-018

Khaniki M: P-045

Khetani K: A-12

Khooamornpongse S: P-064

Kim BM: P-062

Kim DW: P-010, P-011

Kim HK: P-037

Kim HS: P-062

Kim JH: P-001, P-089

Kim MA: P-027

Kim YJ: P-037

Kimura F: P-152

King G: P-178

Kinjo M: P-039, P-040

Kino M: SS-16

Kitabatake N: SS-17

Kitchener H: A-02

Kiyomoto H: P-042

Kjelleberg J: A-20

Klijanienko J: A-42

Klimková M: P-053

Knight B: IL-34

Kobayashi Y: P-113

Kobilkova J: A-53, P-053, P-179

Kochhar S: P-029

Kocjan G: IL-30

Koegelenberg C: A-60

Kok L: A-05

Komatsu A: A-32

Komatsu K: A-32, SS-14

Kon C: P-052

Kondo Y: P-133

Koong SL: P-180

Korfage I: P-168

Korporaal H: A-04, A-07

Koseki M: P-020

Kotaniemi-Talonen L: P-155

Koutselini HG: A-58, A-65, P-098

Koutsky L: IL-04

Kravetz OA: P-054

Kreatsas G: A-64

Krenke R: A-61

Kritvatcharanun K: P-064

Krivak Bolanca I: P-055

Krogerus L: P-028, P-056

Kruger S: A-37

Krzywinski M: IL-20

Kudo M: P-151

Kudoh S: P-052

Kuebler D: A-01

Kugimiya T: SS-16

Kulkarni R: P-047

Kuma S: A-11

Kumar S: P-140

Kuo SH: P-008

Kuraoka K: P-020

Kurjanowa M: P-134

Kurlak M: P-081

Kushida Y: P-042, P-095

Kushima R: P-150

Kusic Z: P-142

Kuwao S: P-152

Kwon JE: P-037
Kyo Young L: P-003, P-114, P-115

Kyriakidou V: A-58, A-65

Kyung Ji L: P-115

Kyungji L: P-114

L

Lachar W: A-25

Lafaras C: P-025

Lai CR: P-180

Lam A: P-132

Lam S: IL-17, IL-24, IL-22

Lam W: IL-22, IL-24

Lama JL: P-171, P-172

Landerer E: A-41

Lau P: P-022

Lau Y: P-164

Laurila P: P-056

Lazarev AF: P-004, P-119

Learmonth GM: A-52, P-030

Lee AK: P-043

Lee D: IL-20

Lee DW: P-010, P-011

Lee JS: P-089

Lee KY: P-009

Lee YT: P-008

Leiman G: A-09, A-38, P-066, P-087, P-121, P-143

Leonardi F: A-57

Li CF: P-080

Li I: IL-20

Li Q: P-106

Liberis V: P-165

Lidang M: A-26

Limoeiro H: P-048

Lin CN: P-080

Lin HP: P-057

Lin O: A-21, P-141

Linder J: SS-13

Ling V: IL-24

Liu YJ: P-008

Lizama A: P-171, P-172

Lo J: P-058

Lo R: P-022

Longatto Filho A: P-070

Losell K: P-035

Loughman N: P-118

Louw M: A-10, A-60, A-62, P-100

Lui P: P-022

Lung-Cheng H: P-059

Lupo C: P-007

Luther A: P-101

Lynch M: P-024, P-026

Lynch MA: P-079

\section{M}

Macaskill P: IL-13

MacAulay C: IL-17, IL-22, IL-23, IL-24

MacDonald S: A-50, A-70

Macias C: A-18

Maeda J: A-22, A-39, P-082, P-097,

P-102, P-103, P-104, P-105, SS-15

Maeda S: P-013, P-116, P-151

Maekawa M: A-11

Mak K: P-022

Mäkisalo H: P-028

Malila N: P-155 
Mamali V: P-170

Mane S: P-044

Maniwa J: P-034

Manojlovic S: P-131

Mantovani M: P-046, P-156

Marra M: IL-20

Martins D: A-18

Marziali A: A-19

Masayuki M: P-049

Masood S: WK-05

Massarani-Wafai R: A-31

Mateaa N: P-142

Mathewson C: IL-20

Matisic J: P-002

Matsui Y: P-015

Matsumoto S: A-46

Matsunaga T: P-042

Matsuta M: P-052

Matsuura Y: P-060

Matthews A: P-143

Matthews R: A-38

Mattosinho de Castro Ferraz MG: P-067, P-068, P-070

May J: P-161

Mayes W: IL-15

McGrath S: P-026

McGuire L: P-132

McIntyre M: A-17

McKenzie P: P-118

McWilliams A: IL-17

Mehrotra R: P-117

Meijer-Marres EM: A-68

Meisels A: IL-07

Meyers T: P-083

Michael C: P-120, P-121

Michelow P: P-083

Mieko MH: P-077

Miller L: P-051

Miller 1: P-161

Miller M: P-007

Mimoto A: P-020

Min K: P-009

Miranda Pereira SM: P-067

Mitchell C: WK-02

Miyahira Y: P-150

Miyajima K: A-22, A-39, P-082, P-102

Miyake S: SS-15

Miyake Y: A-47

Miyauchi A: A-11

Mizugichi K: P-174

Modarres Gilani M: P-045

Modery J: A-48

Moeneclaey L: A-64

Moes G: WK-03

Mohan H: P-029

Montes E: SS-30

Moraes Jr R: P-048

Moravan V: A-28, P-058

Mori I: P-023

Moriuchi A: P-039, P-040

Moriya T: A-47

Mousavi A: P-045

Mukai K: P-103

Munakata S: A-36

Murakami R: P-015

Murali R: P-118
Murata S: P-041

Murata T: SS-17

Murray R: A-37

N

Naito Z: P-013, P-116, P-151

Nakagawa H: SS-18

Nakajima H: P-061

Nakamura M: SS-18

Nakamura Y: SS-17, P-023

Nakanishi K: P-085

Nakanishi Y: A-32, SS-14

Nakao K: P-072

Nakashima N: P-183

Nakayama M: P-107

Nakazawa K: P-041, P-169

Nam JH: P-001, P-089

Nambu M: A-46

Namiyama GM: P-067, P-068

Nanda A: P-029

Nassar A: P-051

Nateewatana S: P-064

Nation J: A-29

Nazari Z: P-045

Nedbalek A: P-185

Nemoto N: A-32, SS-14

Nenci I: A-16, A-43

Neto J: P-014

Ng R: IL-24

$\mathrm{Ng} W$ : P-022

Nga MN: P-043

Nieminen P: P-155

Nishigaki Y: A-56, P-094

Nishimura T: P-020

Nitin N: IL-15

Nizzoli R: A-57

Noamrot P: P-153

Noguchi M: P-072

Nomura M: A-22, A-39, P-082, P-097, P-102, P-104, P-105, SS-15

Norimatsu Y: A-46, A-47, SS-17

Notsu T: P-015

Novosel I: P-127

Numminen K: P-028

Nunobiki O: P-023

Nurani Khojasteh H: P-110

0

Obatake N: P-107

Oh IJ: P-001

Ohira T: A-22, A-39, P-082, P-097, P-102, P-103, P-104, P-105, SS-15

Ohno A: SS-18

Ohno E: A-47

Ohsaki H: A-59, P-042, P-095

Ohyashiki J: A-40

Ohyashiki K: A-40

Oka H: P-088

Okabe H: P-150

Olave E: P-171, P-172

Omori M : P-169

Onda M: P-013

Ono S: P-041

Osada M: P-183

Osamura R: SS-12

Othieno E: A-54
Ouattara O: P-130

Ouwerkerk-Noordam E: A-07, A-67, A-68

Ozaki Y: P-041

Ozbay G: P-033

Ozyegin A: P-145

Pache JC: A-18

Palcic B: IL-14

Palefsky J: IL-10

Panagiotou A: P-112, P-165

Pang Y: P-120, P-121

Pantanowitz L: P-087

Papachristou DJ: P-032

Papadimos D: A-37

Papadopoulou S: P-038

Pappa M: P-098

Parinayok R: P-153

Park CS: P-001, P-089

Park IA: P-027, P-144

Park JY: P-062

Park SM: P-010, P-011

Parra V: P-074, P-108, P-122, P-135

Patel N: P-123

Pavloff N: P-063

Pedriali M: A-16, A-43

Peftoulidis S: P-038

Pei-Yi C: P-059

Pendlebury C: P-164

Pereira SMM: P-068

Peto J: A-02

Pierce M: IL-15

Pierron G: A-42

Pillay KM: A-52

Pinto RGW: P-124, P-125

Pioro P: A-17

Pitman M: WK-02

Plakokefalos E: P-086

Platogiannis D: P-025

Pogacnik A: A-35

Poh C: IL-23

Pohar-Marinsek Z: IL-29

Pokorny J: A-53

Politi EK: A-65

Politi EN: A-58

Polyakova O: P-031

Pothuis P: A-67

Power P: A-29

Prado R: SS-31

Praet M: WK-04, P-073

Preechapornkul N: P-064

Preiszner J: P-051, P-161

Prolla J: P-126

Psachoulia Ch: P-032, P-170

Pu R: P-120, P-121

Pugh T: IL-20

Q

Querzoli P: A-16

$\mathbf{R}$

Raab S: IL-08, IL-33

Ramazanoglu R: P-033, P-145

Ramljak V: P-127

Rana D: P-024, P-026 
Rana DN: P-079

Rangdaeng S: P-064

Ranogajec I: P-127

Rebolj M: A-27, A-30, P-181, P-182

Rehnberg J: A-33

Reinmaa I: P-146

Rekhi B: P-128

Relander T: IL-20

Rerkamnuaychoke B: P-153

Richards A: IL-13

Richards-Kortum R: IL-15

Rijkaart DC: A-68

Rivera M: P-141

Roberts C: A-02

Roberts J: WK-06

Rodil M: P-096

Rodriguez-Costa J: P-074, P-108, P-122, P-135

Rosen D: P-021

Rosin M: IL-23

Ross G: P-002

Rossi S: A-43

Roushdy M: P-149

Roy I: A-54

Rudomina D: A-21

\section{S}

Sabarre A: P-081

Sabry H: P-149

Sachdev A: P-029

Sachiko SM: P-077

Sahebali S: A-06, A-64

Saito M: SS-18

Sakai YI: P-068

Sakamoto N: P-041

Sakman G: P-111

Salhadar A: A-31

Salinero E: P-074, P-108, P-122, P-135

Salva F: P-184

Samija I: P-142

Sanati S: A-44

Sander H: IL-26

Sang-In S: P-114, P-115

Sangkitporn S: P-153

Santini D: A-45

Santos GC: WK-11

Sapardanis J: P-112

Sarasophona S: P-153

Sargent A: A-02

Sarma D: P-106

Sasou S: P-016

Sastre-Garau X: A-42

Sato Y: P-129

Satoh Y: A-55

Satoshi SK: P-077

Satoshi SN: P-077

Satyanarayana S: P-017, P-084

Sauer T: P-018, WK-12

Saville M: IL-11

Sayed H: P-149

Scacchi C: P-007

Scetbon F: A-10

Schein J: IL-20

Schenck U: AW-06

Schleiermacher G: A-42
Schmitt F: IL-28, SS-05

Schneider V: IL-31

Scolyer R: P-118

Seki T: A-32, SS-14

Sentija K: P-055

Serizawa A: SS-12

Serizawa H: SS-15

Settakorn J: P-064

Shariat M: P-045

Shaw H: P-118

Sherman J: A-09, P-066

Sherris J: IL-05

Shiba M: A-15

Shidham V: WK-07

Shim MK: P-001, P-089

Shimajiri S: P-088

Shimizu K: A-47, SS-17

Shimizu M: P-034

Shimizu T: P-094

Shimizu Y: P-034

Shimura M: A-56 P-094

Shin JH: P-009

Shiraishi T: SS-17

Shirata N: P-014

Shirata NK: P-067, P-068, P-070

Shore E: A-24

Siddiqui M: A-66

Siderits R: P-130

Sidira S: P-032

Silva Aguiar L: P-067

Silva C: P-046, P-156

Singh M: P-117

Singh M: P-117

Singh S: P-159

Sioutopoulou D: P-086

Siriunkul S: P-064

Sivakumar S: P-177

Sivaselvam S: P-019

Skagias L: A-65

Slobodan J: A-19

Smith B: A-13, P-081

Smith J: A-23, A-24, SS-04, WK-09

Sneige N: SS-07

Sng KK: P-043

Socias M: A-41, P-171, P-172, P-173

Solding I: P-035

Somrak T: AW-02

Srisomboon J: P-064

St. John T: P-066

Stansfield S: A-37

Steidl C: IL-20

Steinberg W: A-03

Stella CN: P-068

Steben M: IL-03

Stoos-Veic T: P-131

Strigaris K: P-098

Su CC: P-080

Subramanya H: P-084

Sugishima S: P-072

Sugitani M: A-32

Sugiyama Y: SS-16

Sun XR: IL-14, P-006, P-154

Suzuki A: P-085, P-152

Switzer P: A-34

Syrjänen K: A-45, SS-21

Syrjänen S: A-45
T

Tae-Jung K: P-114

Taguchi A: A-15

Tajima Y: P-174

Takahashi Y: P-016

Takatsuka Y: A-36

Takeda A: A-56, P-094

Takeshi TU: P-077

Takeshima N: SS-16

Takeshita M: A-46

Takizawa K: SS-16

Tamiya S: P-072

Tamura G: P-016

Tan SY: P-043

Tan P: P-022

Tang V: P-022

Taniguchi E: P-023

Taniyama K: P-020

Tarkkanen J: P-056, P-155

Tatebe A: P-061

Teramoto S: P-020

Tercelj M: P-002

Terekhova SA: P-004, P-119

Theocharis S: A-42

Theron J: A-62

Thienpont L: P-073

Thompson J: A-19 P-118

Thomson C: A-02

Thomson T: A-13, A-34, P-081

Tiews S: A-03

Tiseo M: A-57

Toda T: P-020

Toki N: P-060

Tomic Brzac H: P-076, P-137

Tominaga S: P-085

Toro V: A-41, P-171, P-172

Trainer T: P-143

Trotman W: A-09, A-38, P-087

Trutin Ostovic K: P-131

Tsay SH: P-180

Tse G: P-022

Tsouma H: A-65

Tsuboi M: P-105

Tsuji K: P-133

Tsukazaki K: SS-18

Tsuyoshi T: P-049

Tsuzuku M: A-55 SS-16, AW-03

Tu HZ: P-154

Tuchiya S: P-013

Turner A: A-02

U

Uchiyama M: P-072

Uematsu M: P-016

Uguz A: P-093, P-111

Urabe R: P-060

Urabe S: P-133

Usuda J: P-097

Utagawa ML: P-070

Utsugi K: SS-16

Uvirova M: P-185

Uygun N: P-033

V

Valente P: A-25 
Valeri R: P-025, P-038, P-086, P-112, P-165

Vamvakopoulos N: P-086

van Ballegooijen M: A-27, A-30, P-181, P-182, P-168

van den Heuvel M: A-62

van Niekerk D: A-28, P-058

Van Velthoven R: P-036

Van Voskuilen I: A-68

Van Wyk C: A-10

Vanden Broeck D: A-06

Vansnick F: P-036

Vanwalleghem L: WK-04

Vasquez JB: P-109

Vass L: A-14

Venkataraman G: A-31

Venter F: A-52

Venter FS: P-030

Vereecken A: A-64, P-157

Verhest A: P-036

Verstovsek G: P-021

Vigil P: P-173

Vilella A: P-184

Villa L: A-41

Villegas J: A-41

Villota C: A-41

Vinkurova S: A-08

Virag M: P-131

von Knebel Doeberitz M: A-08

Vrachliotis T: P-098

Vryenhoef S: P-164

W

Wakasa K: P-107
Wakasa T: P-107, P-023

Walczak C: IL-26

Walker T: P-158

Walsh T: A-37

Wamala DS: P-071

Wang J: IL-14, P-006, P-154

Wang L: A4-3

Wang Y: IL-24

Warren R: IL-20

Wasiutynski A: P-138

Watanabe S: P-072

Watson G: P-118

Weaver D: A-38

Weir M: SS-10

Weiss A: P-162

Weng MH: P-008

Wentzensen N: A-08

Whitehead SEM: P-079

Wilbur D: A-01

Williams M: IL-23

Wing A: P-158

Wohler C: P-172

Wojcik EM: A-17, A-31, P-109

Wong K: IL-20

Wright C: A-10, A-60, A-62, P-083, P-100

Wright G: P-132

\section{$\mathbf{Y}$}

Yada N: P-133

Yamada E: P-150

Yamada H: P-039

Yamamoto K: P-063

Yamamoto LSU: P-067, P-068

Yamamoto Y: A-56, A-59, P-042, P-094

Yamanushi TT: A-59, P-042
Yamawaki T: SS-17

Yamazaki Y: A-56, P-094

Yang G: IL-20

Yanoh K: SS-17

Yarahmadi P: P-090

Yasunaga M: P-072

Yee J: IL-24

Yeong Jin C: P-003

Ylagan L: A-44

Yokoyama K: P-061

Yokoyama M: P-072

Yokoyama Y: P-133

Yonggoo K: P-114

Yoo J: P-009

Yoshida K: A-22, A-39, P-18, P-082, P-097, P-102, P-103, P-104, P-105, SS-15

Yoshida M: P-015

Yoshihito Y: P-049

Yoshino A: A-32

Young N: SS-22

Youn-Soo L: P-114, P-115

Yukihiro YK: P-077

Yuminamochi T: P-041, P-169

\section{Z}

Zarka M: WK-03

Zebrakova I: P-185

Zendehrokh N: A-33, A-40, P-035

Zeren H: P-093, P-111

Zhang L: IL-23

Zhou C: A-28, A-34, A-49, P-058

Ziarkiewicz-Wroblewska B: P-138

Zorludemir S: P-111

Zyli P: P-170 